Modern EPR-related methods in surface science and heterogeneous catalysis*

\author{
Heming, $\mathrm{M}$
}

DOI: https://doi.org/10.1524/zpch.1987.151.part_1_2.035

Posted at the Zurich Open Repository and Archive, University of Zurich ZORA URL: https://doi.org/10.5167/uzh-153927

Journal Article

Published Version

Originally published at:

Heming, M (1987). Modern EPR-related methods in surface science and heterogeneous catalysis*. Zeitschrift für Physikalische Chemie, 151(Part 12$): 35-50$.

DOI: https://doi.org/10.1524/zpch.1987.151.part_1_2.035 


\title{
Modern EPR-Related Methods in Surface Science and Heterogeneous Catalysis*
}

\author{
By M. Heming \\ Physikalisch-Chemisches Institut der Universität, \\ Winterthurerstrasse 190, CH-8057 Zürich, Switzerland
}

(Received July 22, 1986)

\section{Electron spin resonance / Electron nuclear double resonance / Muon spin rotation / Surfaces}

\begin{abstract}
Materials of interest in heterogeneous catalysis are almost exclusively in a polycrystalline or amorphous state. In EPR spectroscopy, orientational averaging, inhomogeneous line broadening and overlap of spectra often lead to a drastic loss of resolution. This might preclude detailed information about the observed species and its interaction with the surroundings. The application of various modern ENDOR and pulse EPR techniques in order to improve the resolution is discussed. In addition, the closely related method of Muon Spin Rotation ( $\mu \mathrm{SR})$ is considered in view of its possible contributions to the study of Muonium, the light isotope of hydrogen, and muonium-labeled organic free radicals on surfaces.
\end{abstract}

Katalytisch interessante Materialien liegen häufig nur in polykristalliner oder amorpher Form vor. Dementsprechend sind die zugehörigen EPR-Spektren in der Regel als Folge der räumlichen Mittelung sowie einer starken inhomogenen Linienverbreiterung und eventuell einer Überlagerung verschiedener Spektren nur schlecht aufgelöst. Die Anwendung verschiedener ENDOR- und EPR-Puls-Methoden zur Verbesserung des Auflösungsvermögens wird diskutiert. Zusätzlich wird die Myonen-Spin-Rotations-( $\mu$ SR)Technik diskutiert. Mittels dieser Methode können Myonium (das leichte Isotop des Wasserstoffatoms) und Myonium-substituierte Radikale auf Oberflächen untersucht werden.

\section{Introduction}

Within the last 30 years EPR has become a powerful and indispensible tool for the study of paramagnetic species in heterogeneous catalysis and surface science $[1-5]$. In principle EPR is capable of delivering information concerning the nature of paramagnetic species, their electronic and

* Presented as a Plenary Lecture at the IV. International Symposium on Magnetic Resonance in Colloid and Interface Science, Münster, FRG, July 21 - 25, 1986. 
geometrical structure, their interaction with the surroundings, their reactivity and kinetics. An accurate characterization of a paramagnetic species and of the interaction with its surroundings is a formidable task. It requires the determination of as many as possible Spin-Hamilton parameters, including the orientation of the principle axes of the various tensors. In addition a careful analysis of the lineshape is necessary to elucidate dynamical aspects $[6,7]$, and kinetic studies may involve time-resolved EPR techniques on various levels of sophistication. An ideal EPR experiment therefore requires a well-defined system, e.g. a single-crystal surface. Although very few experiments have been carried out on such systems (e.g. [8]) demonstrating the capacities of EPR spectroscopy, the overwhelming majority of materials in heterogeneous catalysis is in a polycrystalline or, even worse, amorphous state. Therefore, the determination of the orientation of the principal axes of the various tensors is a priori not possible. Orientational averaging of the anisotropic interactions, distribution of Spin-Hamilton parameters rather than well-defined values, and inhomogeneous line broadening as a consequence of weak dipolar interactions often result in a poorly resolved EPR spectrum and make a meaningful quantitative analysis difficult, if not impossible. Additional complications may arise through extensive overlap of spectra of different species.

In the case of inhomogeneous line broadening a considerable resolution enhancement can be achieved by applying techniques, which are capable of directly measuring NMR transition frequencies in paramagnetic systems. It is the purpose of this paper to discuss with this respect the possible applications of more advanced EPR techniques, like the various forms of Electron Nuclear Double Resonance (ENDOR) spectroscopy [9-11] and Electron Spin Echo (ESE) spectroscopy $[12,13]$, the pulse variant of $\mathrm{cw}$ EPR. In addition, the closely related method of Muon Spin Rotation $(\mu S R)$ [14] will be discussed in view of its potential contributions to the study of Muonium, the light isotope of hydrogen, and muonium-labeled organic radicals on surfaces.

\section{Electron nuclear double resonance}

In an ENDOR experiment one simultaneously performs an EPR and a NMR experiment by observing the effect of a strong radio frequency (rf) field on a partially saturated EPR absorption. The technique and its applications have been extensively covered in recent monographs [9-11].

ENDOR on materials in a disordered state may give rise to two different types of spectra $[9,15]$ : (1) anisotropic ENDOR, i.e. frequencies do occur displaced from the free nuclear precession frequency, and (2) Matrix ENDOR, which often constitutes of a more or less structured signal centered at the nuclear Larmor frequency. 


\subsection{Anisotropic ENDOR}

The shape of the spectrum depends characteristically on the part of the EPR spectrum which is saturated, on the degree and kind of anisotropy and on the dominant relaxation pathways. It is the latter fact, which may complicate a simulation of the lineshape considerably.

Rist and Hyde [16, 17] first pointed out the possibility to obtain ENDOR spectra which correspond only to a limited set of orientations, if the ENDOR effect is observed at the turning points of an EPR spectrum. Specifically, they noted that it is possible to obtain single-crystal type spectra for systems where

(1) one magnetic interaction (hyperfine anisotropy, zero-field splitting or g-anisotropy) dominates the EPR spectrum or

(2) two magnetic interactions have a comparable degree of anisotropy, but the axes of largest anisotropy coincide.

As an example of (2), the EPR and ENDOR spectra of $\mathrm{VO}^{2+}$ adsorbed on $\mathrm{NaY}$ [18] are shown in Fig. $1 \mathrm{a}, \mathrm{b}$. The zeolite is in a fully hydrated state. In the EPR spectrum no hyperfine coupling with neighbouring nuclei is observed. With the magnetic field set at the $m_{\mathrm{I}}=-7 / 2_{\|}$transition, only orientations along the common $z$-axes of the $\mathrm{g}$ - and the $\mathrm{VO}^{2+}$-hyperfine tensor are selected. The ENDOR spectrum is readily identified to be due to $\mathrm{VO}\left(\mathrm{H}_{2} \mathrm{O}\right)_{5}^{2+}$ by comparison with the frozen solution spectrum. The frequencies correspond to the hyperfine tensor components of the "axial" and "equatorial" protons along the $\mathrm{V}=\mathrm{O}$ bond direction. On the other hand, a quasi two-dimensional spectrum is obtained with the magnetic field set at the $M_{1}=-3 / 2_{\perp}$ transition, as shown in Fig. $1 \mathrm{c}$. In addition to
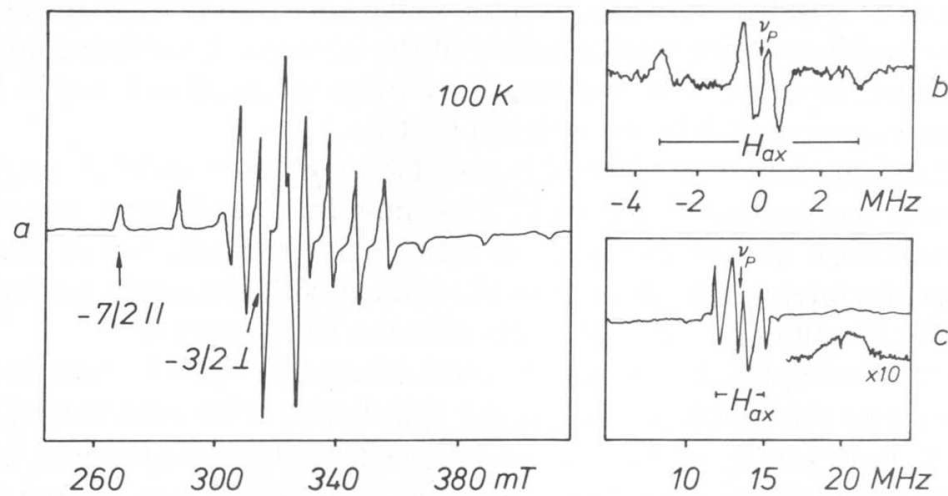

Fig. 1. (a) EPR spectrum of the $\mathrm{VO}\left(\mathrm{H}_{2} \mathrm{O}\right)_{5}^{2+}$-complex in fully hydrated $\mathrm{NaY}$. A singlecrystal like ENDOR spectrum (b) is observed with the magnetic field set at the $m_{\mathrm{l}}=$ $-7 / 2_{\|}$transition. For the magnetic field set at the $m_{1}=-3 / 2_{\perp}$ transition a predominantly two-dimensional ENDOR spectrum (c) is obtained. Signals due to protons in the axial water molecule are denoted as $\mathrm{H}_{\mathrm{ax}} \cdot v_{\mathrm{p}}$ markes the free nuclear precession frequency. No Matrix-line is observed. Redrawn from Ref. [18] with permission of the author. 
the perpendicular (relative to the $\mathrm{V}=\mathrm{O}$ bond direction) hyperfine tensor components of the axial protons, the spectrum reveals the parallel (very weak) and perpendicular components of the equatorial protons. Evacuation at room temperature leads to a loss of the proton signals, which has been interpreted in terms of binding of $\mathrm{VO}^{2+}$ to four oxygens of the zeolite network and loss of the axial water molecule.

In order to obtain the true principal values of the hyperfine tensor it is necessary to record the ENDOR spectrum over the entire EPR spectrum $[19,20]$. A theory of polycrystalline ENDOR patterns for dominant ganisotropy [19] reveals that it is not only possible to extract the principal values of the hyperfine tensor, but that in addition the relative orientations of the g- and A-tensors may be deduced from extra divergences and maxima in the ENDOR spectrum at intermediate field settings.

Alternatively, some of the principal values of the hyperfine tensor and the orientations of their principal axes may be obtained from ENDORinduced EPR spectra [21]. The idea is to measure the changes of the amplitude of an ENDOR frequency $v=v_{\mathrm{n}}+\Delta v$ as a function of the magnetic field. For a single coded (frequency modulation only) ENDOR line the EPR spectrum is readily obtained in the form of an absorption spectrum. For $\Delta v=1 / 2 A_{\max (\min )}$, a single-crystal type EPR spectrum is obtained. Since the frequency is field dependent one has to adjust the rffield whilst scanning the magnetic field.

In contrast to the orientation selected ENDOR spectra, powder type spectra are obtained for arbitrary magnetic field settings or whenever conditions (1) and (2) are not fullfilled. In general, the ENDOR intensity will be the better the lower the hyperfine anisotropy is. Thus, $\beta$-couplings of organic free radicals are usually quite well resolved since their anisotropy constitutes often only a small fraction of the isotropic hyperfine coupling. In contrast, signals due to $\alpha$-protons are often smeared out over a large frequency range and difficult to detect $[9,15]$.

Indeed, the ease with which it is possible to measure small $\beta$-couplings accurately has enabled Clarkson [22] to show that the $\beta$-couplings of the perylene cation radical adsorbed on metal oxide surfaces can be used to monitor the interaction strength of the radical with the acidic adsorption sites. The $\beta$-couplings were not resolved in the EPR spectra.

So far, we implicitly assumed the cross relaxation rate $\left(T_{x}^{-1}\right)$ to be slow compared to the electron and nuclear spin-lattice relaxation rates $\left(T_{1 \mathrm{e}}^{-1}\right.$, $\left.T_{1 \mathrm{n}}^{-1}\right)$. If, however, $T_{x} \ll T_{1 \mathrm{e}}, T_{1 \mathrm{n}}$ a true powder pattern is obtained, i.e. all possible orientations contribute irrespective of which part of the EPR spectrum is saturated. This case has been treated by Dalton and Kwiram [23]. Under this condition ENDOR-induced EPR may be used to separate overlapping EPR spectra.

In the last few years Schweiger and coworkers introduced a number of advanced techniques with the aim to simplify complicated single-crystal 
spectra. Among them, ENDOR utilizing a circular-polarized (CP-ENDOR) [24] or a polarization-modulated (PM-ENDOR) [25] rf-field should turn out to be useful for the interpretation of anisotropic spectra too.

In CP-ENDOR one takes advantage of the fact, that the transition probability induced by the left hand (l.h.) or right hand (r.h.) rotating rffield component depends on the relative magnitude of the external magnetic field $B_{\mathrm{o}}(\uparrow)$ and the internal magnetic field $B_{\mathrm{e}}(\hat{\uparrow})$. If $B_{\mathrm{e}}$ outweights $B_{\mathrm{o}}$ than the direction of the effective magnetic field $B_{\text {eff }}(\Uparrow)$ changes its sign with $m_{\mathrm{s}}$ :

$$
m_{\mathrm{s}}=+1 / 2: \uparrow+=\mathbb{W} \quad m_{\mathrm{s}}=-1 / 2: \uparrow+\hat{\mathbb{1}}=\mathbb{\$} .
$$

For this simplified example of isotropic couplings $\left(a, g_{n}>0\right)$ only r.h. (l.h.) rotating field components induce transitions for $m_{\mathrm{S}}=+1 / 2(-1 / 2)$.

On the other hand, in PM-ENDOR a rotating rf-field causes a modulation of the transition amplitude. The modulation depth decreases with the hyperfine interaction. Thus, PM-ENDOR is a technique suitable to suppress the Matrix-line.

\subsection{Matrix-ENDOR}

Besides the anisotropic lines a common feature in the spectra of disordered materials is the so-called Matrix-line [26] occurring at the free nuclear precession frequency. This line is due to weakly coupled nuclei, the main interaction being the electron-nuclear dipolar interaction. As an example, the Matrix-line originating from the interaction between the $V_{\mathrm{OH}}^{*}$ surface defect centre in the $\mathrm{MgO}(111)$ crystal face and physisorbed $\mathrm{H}_{2}$ at $4.2 \mathrm{~K}$ is shown in Fig. 2 [27]. An average interaction distance of $\langle r\rangle=0.52 \mathrm{~nm}$ has been inferred from the line-width.

A quantitative analysis of the lineshape following the ideas of Hyde and coworkers $[26,28]$ is, in general, tedious and does not always provide a unique set of parameters [15]. The lineshape is particularly sensitive to the nuclear relaxation mechanism. Given a $S=1 / 2, I=1 / 2$ spin system a Pake doublett would be expected for an isotropic nuclear relaxation rate $T_{1 \mathrm{n}}^{-1}$. However, assuming that the nuclear relaxation occurs primary through the modulation of the electron-nuclear dipolar interaction via the relaxing electron spin, $T_{1 \mathrm{n}}^{-1}$ becomes orientation dependent and the doublett merges into a singulett [26]. Within the last few years it turned out that the analysis of the modulation effect in ESE spectroscopy is better suited to obtain structural informations from very weakly coupled nuclei $[12,13]$.

\section{Electron spin echo (ESE) spectroscopy}

In an ESE experiment one monitors the spontaneous outburst of microwave energy as a function of the timing of two or more short resonant microwave 


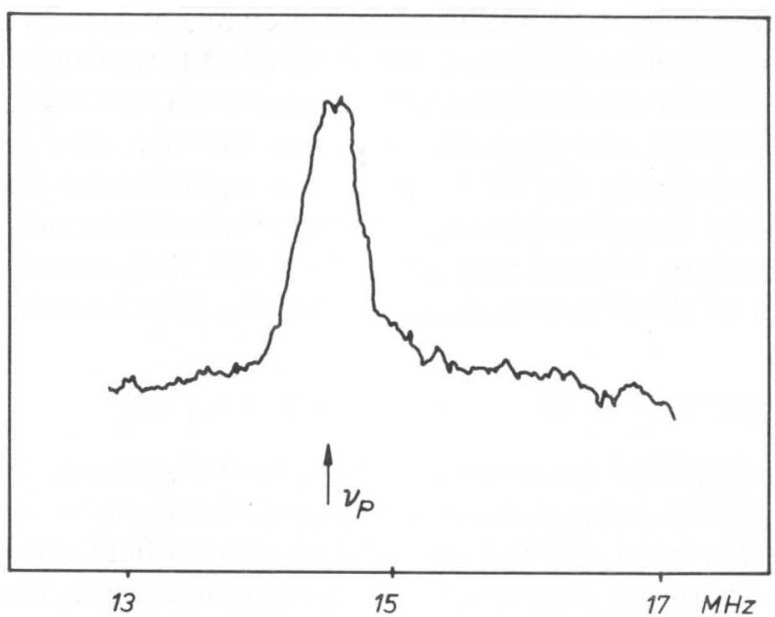

Fig. 2. ENDOR signal of the $V_{\mathrm{OH}}^{*}$ surface centre in $\mathrm{MgO}$ after adsorption of $\mathrm{H}_{2}$ at $4.2 \mathrm{~K}$. Redrawn from Ref. [27] with permission of the author.

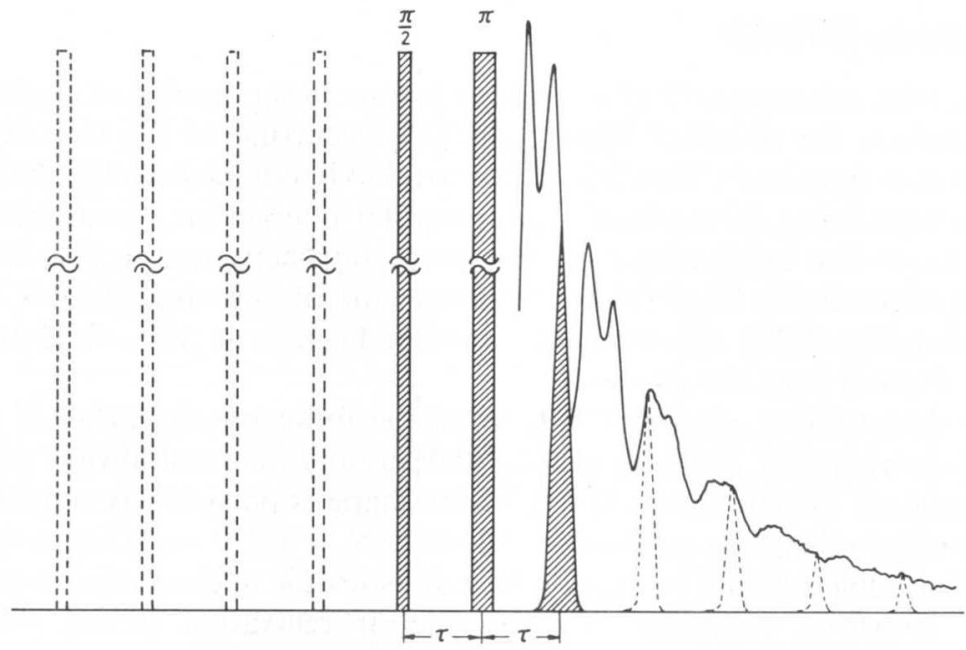

Fig. 3. Schematic of the 2 pulse Electron Spin Echo Modulation experiment.

pulses. In a two-pulse experiment, the initial $\pi / 2$ pulse prepares the spin system in a coherent state. Subsequently, the spin packets, each characterized by its own Larmor precession frequency $\omega_{i}$, start to dephase. A second $\pi$-pulse at time $\tau$ effectively reverses the time evolution of the spin packet magnetizations, i.e. the spin packets start to rephase, and an emission of microwave energy (the primary echo) occurs at time $2 \tau$. The echo amplitude as a function of $\tau$ constitutes the ESE spectrum (Fig. 3). 
Relaxation processes lead to an irreversible loss of phase correlation, thus the amplitude decays with a characteristic time $T_{2}^{\mathrm{M}}$, the phase memory time. Fortunately, the decay is often accompanied by a modulation of the echo amplitude. Analysis of the modulation frequencies and amplitudes forms the basis of the Electron Spin Echo Modulation (ESEM) spectroscopy. The modulation effect is a consequence of the violation of the high field approximation. Non-diagonal elements due to anisotropic hyperfine and quadrupole interactions cause mixing of nuclear levels. This in turn allows the simultaneous excitation of allowed and forbidden transitions, if the microwave magnetic field is strong enough. The branching of transitions causes interferences, which manifest themselves as a modulation of the echo amplitude. A convenient expression in terms of the eigenfunctions and eigenvalues of a spin system under the assumption of complete excitation of the EPR spectrum has been given by Mims $[29,30]$. For a $S=1 / 2, I=1 / 2$ system the modulation function can be written as [29]:

$$
\begin{aligned}
& V_{\bmod }(2 \tau)= 1-k / 4\left[2-2 \cos \omega_{\alpha} \tau-2 \cos \omega_{\beta} \tau\right. \\
&\left.+\cos \left(\omega_{\alpha}-\omega_{\beta}\right) \tau+\cos \left(\omega_{\alpha}+\omega_{\beta}\right) \tau\right] \\
& k=\omega_{\mathrm{I}} B / \omega_{\alpha} \omega_{\beta}, \quad B=g g_{\mathrm{n}} \beta \beta_{\mathrm{n}}(3 \sin \theta \cos \theta) / \hbar \mathrm{r}^{3}
\end{aligned}
$$

$\omega_{\alpha, \beta}$ are the ENDOR frequencies, $\omega_{1}$ is the nuclear Zeeman frequency, $\theta$ the angle between the magnetic field $B_{0}$ and the electron-nuclear axis. All other symbols have their usual meaning in magnetic resonance. In deriving Eq. (1) the validity of the point-dipole approximation and an isotropic gfactor have been assumed.

The frequency spectrum contains not only the ENDOR frequencies, but in addition their sums and differences. For weakly coupled nuclei, $\omega_{x, \beta}$ $\cong \omega_{\mathrm{I}}$, and only two frequencies are observed. In the presence of $n$ interacting nuclei the total modulation function is simply the product of the individual modulation functions $[29,30]$ :

$$
V_{\bmod }^{\mathrm{n}}=\Pi_{i} V_{\bmod }^{i} .
$$

From Eqs. (1) and (2) it is evident that the modulation depth increases strongly with the number of interacting nuclei and a decreasing electronnuclear distance. In addition, the modulation depth also increases with the nuclear spin $I$ [29]. However, it has to be recalled that close and therefore strongly interacting nuclei are ESE-silent. In disordered matrices a simulation of the ESE spectrum requires, in principle, a geometrical model for the mutual arrangement of the interacting nuclei. On the other hand it is the aim of the experiment to establish such a model. Consequently, Kevan et al. [31] argued to base the data analysis on a spherically uncorrelated distribution model, i.e.

$$
V_{\text {mod }}^{\mathrm{n}}=\left[<V_{\bmod }\left(r, a_{\text {iso }}\right)>\grave{\theta}\right]^{\mathrm{n}}
$$




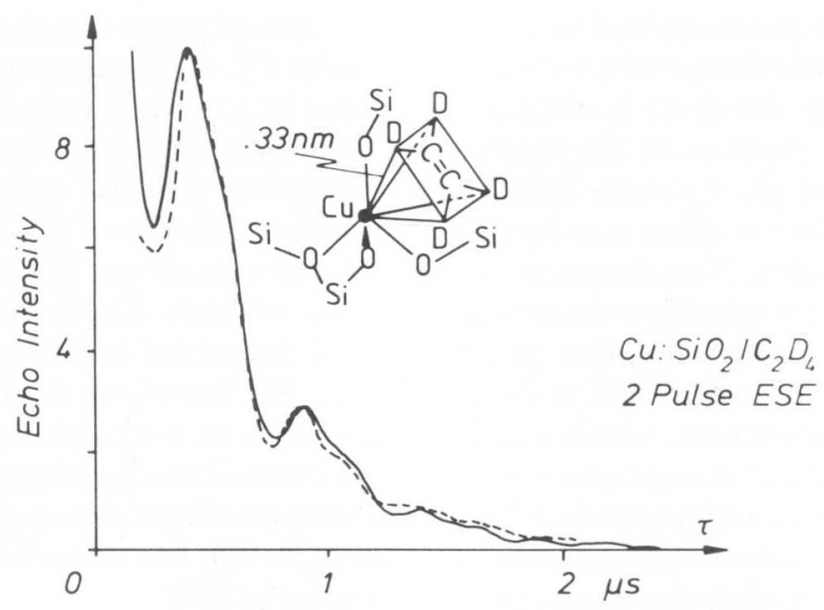

Fig. 4. Calculated $(\longrightarrow$ ) and experimental (---) 2 pulse ESEM spectra for impregnated $\mathrm{Cu}: \mathrm{SiO}_{2}$ activated at $1000 \mathrm{~K}$ and with adsorbed $\mathrm{C}_{2} \mathrm{D}_{4}$. The proposed model for the $\mathrm{Cu}^{2+}-\mathrm{C}_{2} \mathrm{D}_{4}$ interaction is shown too. Redrawn from Ref. [33] with permission from L. Kevan.

where $r$ is an effective shell distance. This model is now widely accepted [32]. A fit reveals three parameters, the number of interacting nuclei, their effective distance and the Fermi contact interaction.

As an example the two-pulse ESE spectrum for impregnated $\mathrm{Cu}^{2+}$ in a silica gel activated at $1000 \mathrm{~K}$ with subsequently adsorbed deuterated ethylene is shown in Fig. 4 [33]. The analysis indicates four deuterons at $r=0.33 \mathrm{~nm}$ and with $a_{\text {iso }}=0.15 \mathrm{MHz}$. At this distance a direct bond between $\mathrm{Cu}^{2+}$ and ethylene is clearly excluded. Together with the information derived from the g-factors, the authors were able to propose a detailed geometrical model, which is shown in the same figure. In essence, the $\mathrm{Cu}-$ $\mathrm{O}_{4}$ tetrahedra distort upon adsorption of ethylene towards either a trigonalbipyramidal or a square-pyramidal geometry.

Instead of stepping $\tau$ from experiment to experiment the full information can be obtained in a single step by replacing the initial $\pi / 2$-pulse with a low-level extended-time excitation, e.g. a cw-microwave magnetic field with constant amplitude and appropriate frequency [34]. However, one has to take some sensitivity loss into account.

The main limitation of the two-pulse experiment originates from phase relaxation processes. If $T_{2}^{\mathrm{M}}$ becomes too short the instrumental dead time, typically about $150 \mathrm{~ns}$, may cover a substantial part of the ESE spectrum. Further, multiple frequencies complicate the spectrum. Both problems may be overcome by observing the stimulated echo in a three-pulse sequence. Dividing the second pulse of the two-pulse experiment into two $\pi / 2$ pulses 


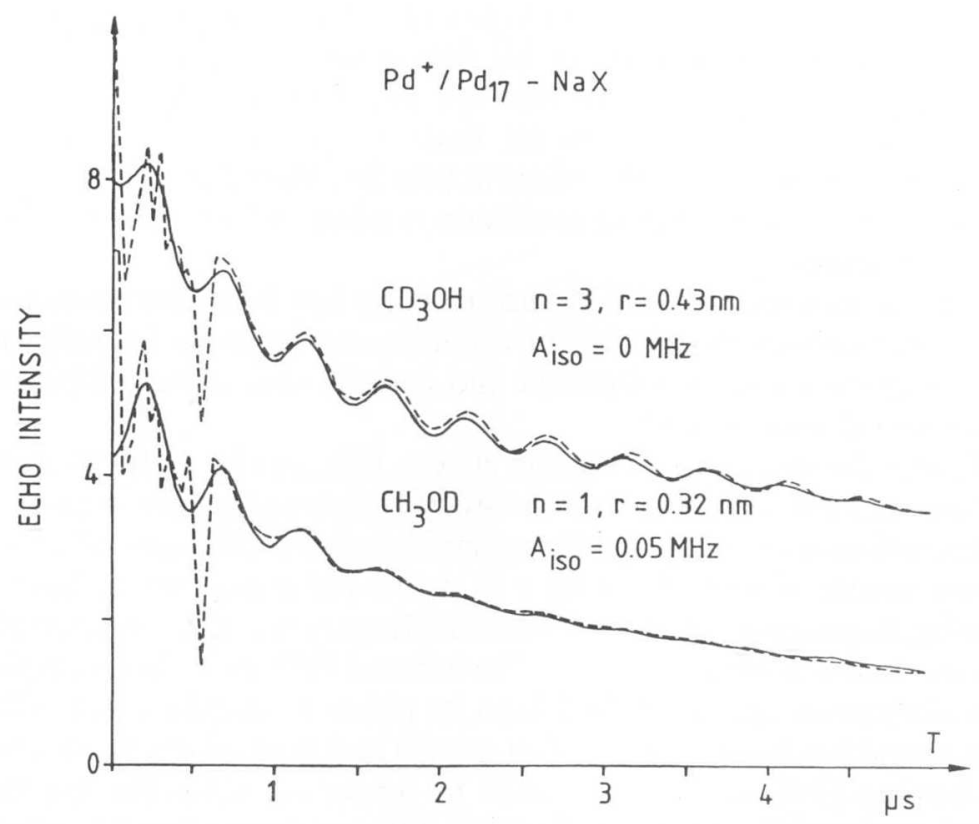

Fig. 5. Calculated (-) and experimental (----) 3 pulse ESEM spectra for $\mathrm{Pd}^{+} / \mathrm{Pd}_{17^{-}}$ $\mathrm{NaX}$ formed after methanol adsorption. Upper: $\mathrm{CD}_{3} \mathrm{OH}$, lower $\mathrm{CH}_{3} \mathrm{OD}$.

essentially stores the magnetization in the $-z$ direction. The echo can now be followed over a time of the order of magnitude $T_{1} \gg T_{2}^{\mathrm{M}}$. With the separation between the first and the second pulse denoted as $\tau$ and the one between the second and third as $T$, the stimulated echo, occurring at $2 \tau+$ $T$, is monitored in the $T$-space. The modulation function can be calculated from Eq. (1) [30]:

$$
V_{\text {mod }}^{3 \text { p }}=1 / 2\left[V_{\alpha}(\tau, T)+V_{\beta}(\tau, T)\right] .
$$

In contrast to the two-pulse modulation function the sums and differences of the ENDOR frequencies are now eliminated. Maximum modulationoccurs for weakly interacting nuclei for $\omega_{1} \tau=(2 n+1) \pi$, whereas for $\omega_{1} \tau=2 \mathrm{n} \pi$ the modulation amplitude vanishes. In general, the threepulse experiment is superior to the two-pulse experiment. However, several experiments with different $\tau$ settings have to be performed to make sure that no frequencies are overlooked. Alternatively, the proper selection of $\tau$ may suppress unwanted frequencies and simplify the analysis.

Fig. 5 shows the three-pulse ESE spectrum of $\mathrm{Pd}^{+}$in $\mathrm{Pd}_{17}-\mathrm{NaX}$ formed after methanol adsorption [35]. Selectively deuterated methanol was used in order to determine the orientation of the adsorbate with respect to $\mathrm{Pd}^{+}$. $\tau$ was chosen such as to suppress ${ }^{27} \mathrm{Al}$-modulation. The analysis indicates 
interaction with one methanol molecule and a molecular dipole orientation. $\mathrm{Pd}^{+}$is probably located at SII' or SII sites in the sodalite cages.

Several glitches in the spectrum are apparent and disturbing. They originate from two-pulse echoes (at times $T=0$ and $T=\tau$ ) and from a refocussing echo with opposite phase at time $2 \tau$. Recently, Fauth et al. [36] demonstrated a phase-cycling excitation method, which eliminates these unwanted echoes.

The full potential of ESEM spectroscopy has been demonstrated by Kevan and coworkers $[37,38]$ in a continuing series of investigations, concerning the location, migration and coordination of transition metal ions on metal oxide surfaces.

Besides the study of modulation effects, ESE can be useful to sort out overlapping EPR spectra and for the study of slow motional processes. For the separation of overlapping EPR spectra one takes advantage of the timedomain nature of ESE. Keeping $\tau$ in a two-pulse experiment fixed and sweeping $B_{0}$ produces an ESE-induced EPR spectrum as long as the phase memory time is field independent. The induced EPR spectrum is displayed as an absorption spectrum. $B_{1}$ has to be reduced compared to ESEM to allow a good resolution of the EPR spectrum and to avoid any modulations. Overlapping EPR spectra may easily be separated, if the two (or more) paramagnetic species exhibit sufficiently different phase-memory times, by proper selection of $\tau$. On the other hand, significantly slower motions can be studied by ESE, since the phase memory time can be several orders of magnitude higher than $T_{2}^{*}$, the inverse of the inhomogeneously broadened EPR line. In addition, the spin packet line-width can be monitored over the entire EPR spectrum in a two-dimensional experiment providing very detailed information about motional processes [39].

\section{Muon spin rotation}

Although chemists have appreciated for a long time the role of elementary particles, such as the electron, the proton or the neutron, in studies concerning the structure and dynamics of matter, other elementary particles are still regarded as being to some extent "exotic".

To begin with, it therefore seems appropriate to introduce in brief the positive muon and to emphasize its usefullness as a spin probe from the point of view of a chemist [40].

The positive muon is produced in the parity-violating decay of a positive pion into a muon and a neutrino:

$$
\pi^{+} \rightarrow \mu^{+}+v_{\mu} \text {. }
$$

Like the proton it is a spin $1 / 2$ particle. However, its associated magnetic moment is 3.18 times larger than the proton magnetic moment, whereas its mass is smaller by a factor of 9 . It is a short-lived particle $\left(t_{1 / 2}=1.5 \mu \mathrm{s}\right)$ 
and decays into a positron and two neutrinos. When a muon is stopped in matter it thermalizes within less than $1 \mathrm{ps}$. During this time it can associate with an electron. Thus, $\mathrm{Mu}\left(\equiv \mu^{+} \mathrm{e}^{-}\right)$is formed, which should be regarded as the light isotope of hydrogen $\left(\equiv p^{+} \mathrm{e}^{-}\right)$. Indeed, the Bohr radius, the reduced mass and the ionization energy coincide within less than $0.5 \%$. As an isotope of $\mathrm{H}, \mathrm{Mu}$ behaves chemically (besides a sometimes hughe isotope effect) very much alike. Mu can undergo a variety of reactions, including the addition to an unsaturated bond:

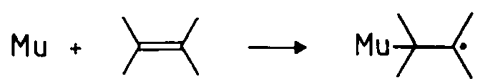

Thereby a muonated radical is formed.

Muonium and muonated radicals have been mainly studied by the timedomain technique of Muon Spin Rotation ( $\mu \mathrm{SR})$. This technique has been very successfully exploited for [41]:

(1) Studies of the kinetics of $\mathrm{Mu}$ reactions in the gas phase and the liquid phase. Especially, the gas phase studies have provided a very critical testing ground for reaction rate theories.

(2) The determination of absolute rate constants of fast organic radical reactions in the liquid phase. In this case the muon is used as a passive spin probe.

The $\mu$ SR-technique is outlined in Fig. 6. Spin-polarized muons are stopped in a probe, which itself is placed in a magnetic field transverse with respect to the spin polarization of the incoming muons. The muon spin then starts to precess with a frequency depending characteristically on the locally experienced magnetic field. The evolution of the muon spin polarization in time and space is monitored by measuring the time, which the muon spends in the probe until it decays, and the direction of the emitted positron. The latter is preferably emitted along the instantaneous direction of the muon spin. The technique requires that only one muon at a time is present in the probe. Summing up events (typically a few million) for a given direction yields radioactive muon decay curves, modulated by muon spin precession frequencies. Analysis of these curves in the Fourier space [42], reveals these frequencies (hyperfine couplings), their amplitudes (yields), their phases (information about the formation history) and their relaxation rates (incl. chemical reaction rates). Essentially, the technique parallels an ideal FT-NMR experiment, where one would monitor the FID after an infinitely strong $90^{\circ}$-pulse.

Like EPR, $\mu \mathrm{SR}$ is not a surface specific technique. The main obstacle is, of course, to thermalize sufficient muons outside the bulk material. It has been firmly established that, by using a $7 \mathrm{~nm}$ diameter non-porous silica powder as a probe, the muons almost exclusively thermalize as $\mathrm{Mu}$ in the void regions between the powder grains [43]. A negative work function may be responsible for this observation [44]. Fig. 7 shows the relaxation rate for $\mathrm{Mu}$ on a silica surface as a function of temperature [45]. Below 


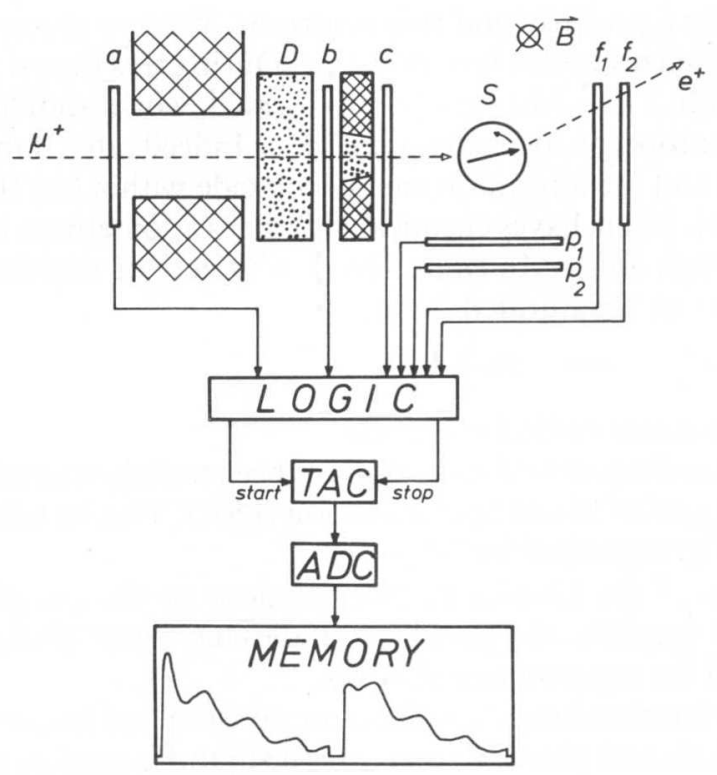

Fig. 6. Schematic of the time-differential $\mu \mathrm{SR}$ experiment. $S$ : sample, $a, b, c, f_{1}, f_{2}, p_{1}$, $p_{2}$ : scintillation counters, $D$ : degrader, $B$ : magnetic field. Reproduced from Ref. [41] with permission of the author.

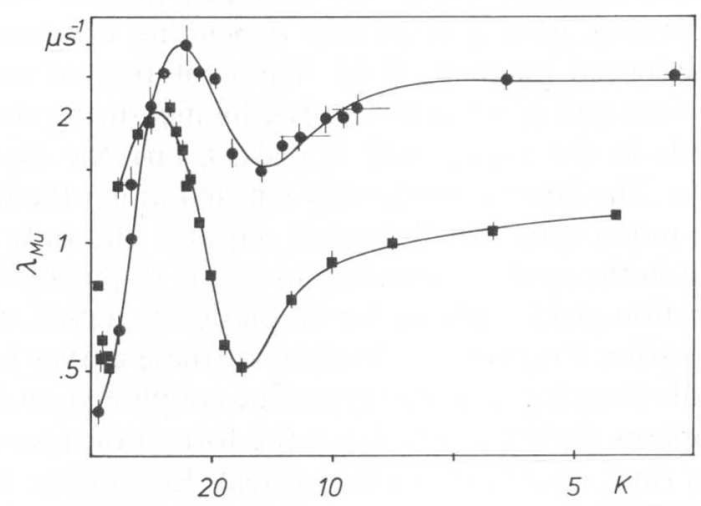

Fig. 7. Mu relaxation rate on a silica surface in a transverse magnetic field of $7.3 \cdot 10^{-4} \mathrm{~T}$. - $\mathrm{SiO}_{2}$ evacuated at $383 \mathrm{~K}$; $\mathrm{SiO}_{2}$ evacuated at $873 \mathrm{~K}$. Redrawn from Ref. [45] with permission of the author.

$8 \mathrm{~K}, \mathrm{Mu}$ is localized on the surface, the relaxation rate being mainly caused by dipolar interactions with the surface hydroxyls. Above $8 \mathrm{~K}$, Mu starts to move on the surface and motional narrowing decreases the relaxation rate, until $\mathrm{Mu}$ becomes localized in deeper traps at $25 \mathrm{~K}$. From longitudinal decoupling experiments it has been argued that these traps are due to $\mathrm{Fe}^{3+}$ 


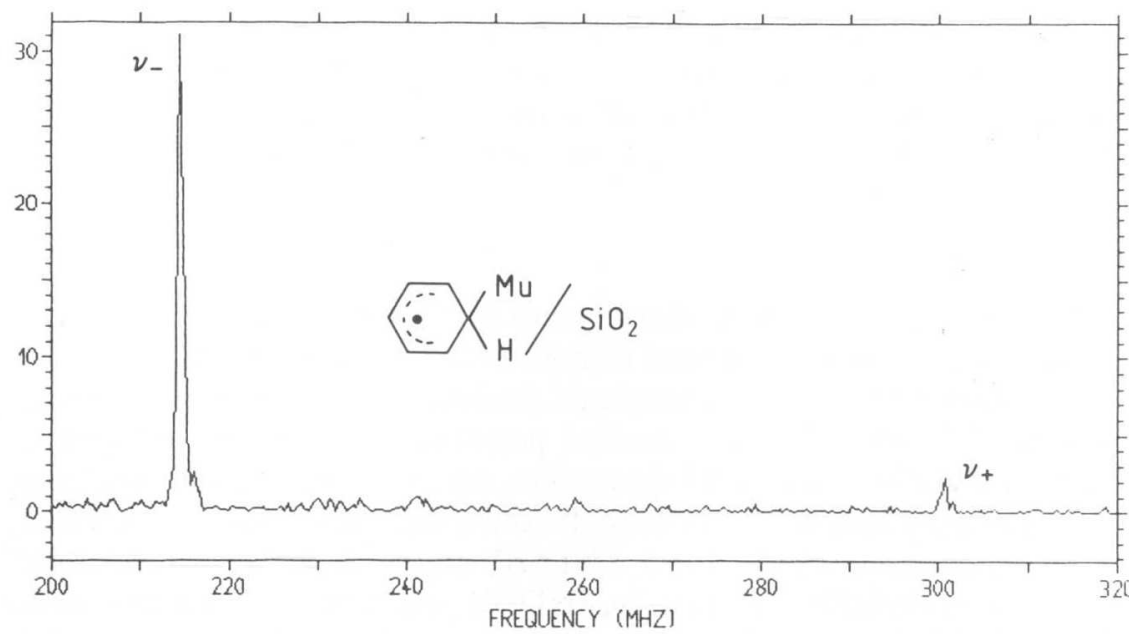

Fig. 8. Fourier power spectrum of the muonated cyclohexadienyl radical on a fully hydroxylated $\mathrm{SiO}_{2}$ surface at room temperature in a magnetic field of $0.238 T$.

impurities (about $6 \mathrm{ppm}$ ). At still higher temperatures detrapping and eventually desorption of Mu occurs.

In addition to the reaction kinetics of $\mathrm{Mu}$ with ethylene on a silica surface [46], the reaction of $\mathrm{Mu}$ with a platinum-loaded silica surface has been studied. The Pt-crystallites were covered by oxygen and a steep increase of the relaxation rate around $40-60 \mathrm{~K}$ has been interpreted in terms of a chemical reaction $[47,48]$ :

$$
\mathrm{Mu}+\mathrm{O}(\mathrm{ads}) \rightarrow \mathrm{OMu} \text {. }
$$

Recently, we succeeded to observe a muonated radical on a surface [49]. Using a hydroxylated silica surface, which was covered by appr. a monolayer of benzene, we were able to detect the cyclohexadienyl radical. The Fourier power spectrum is shown in Fig. 8. Like in ENDOR spectroscopy, two frequencies are observed, and their sum equals the hyperfine coupling constant $A_{\mu}$. A large difference in the amplitudes of the two frequencies is evident. Due to the finite lifetime of the $\mathrm{Mu}$ precursor (compared to the Mu precession frequency), the muons do not enter the product state (the radical) coherently. The resulting depolarization depends on the muon precession frequencies in the precursor, in the product state and on the formation rate [50]. The simplicity of the spectrum is remarkable and it should be possible to study the behaviour of muonated radicals on surfaces. In order to measure the other nuclear hyperfine couplings one has to resort to the recently proposed [51] and developed [52, 53] technique of Avoided Level Crossing $\mu \mathrm{SR}$ in longitudinal magnetic fields. 
Although the possibilities of $\mu \mathrm{SR}$ in surface science are quite limited today, this situation will certainly improve in the near future, when near thermal $\mu^{+}$- or Mu-beams become available. There is now considerable effort at the major meson factories to construct such beam lines.

\section{Comparison}

Although all three techniques directly measure NMR transition frequencies in paramagnetic systems, several distinct differences are apparent.

In disordered systems a lineshape analysis is, of course, most easily performed in $\mu \mathrm{SR}$. No instrumental perturbations (e.g electromagnetic fields, modulation effects etc.) are present, and, in addition all orientations of the paramagnetic species contribute to the lineshape. This is in contrast to the orientation selectivity in ENDOR and ESEM due to the finite excitation of the EPR spectrum. In ENDOR orientation dependent transition probabilities arise via the rf-enhancement effect and orientation dependent relaxation pathways. In ESEM it is the modulation effect itself which is strongly orientation dependent. E.g. neglecting quadrupole interactions, the modulation effect vanishes in a point-dipole model for $B_{0}$ parallel or perpendicular to the electron-nuclear axis. Thus, for weakly interacting nuclei often only a bell-shaped signal is observed $[54,55]$.

It is important to note, that the techniques monitor different regions of the spin density distribution. ESEM mainly operates in the low frequency region. The branching of transitions condition and the finite timing resolution imposes an upper limit of appr. $30 \mathrm{MHz}$. On the other hand, it is difficult to obtain an ENDOR response from weakly interacting nuclei with low gyromagnetic ratios due to difficulties in saturating the NMR transitions. Likewise it is difficult to measure weak nuclear hyperfine frequencies in $\mu \mathrm{SR}$ [53]. However, the timing resolution is much better than in ESEM and muon hyperfine frequencies of $>4 \mathrm{GHz}$ have been measured [56].

Compared to ENDOR the advantages of ESEM are: (1) the normalized modulation amplitude is solely determined by Spin-Hamilton parameters, including the number of interacting nuclei. (2) ESEM is not necessarily less sensitive than EPR, an ENDOR response is commonly one to two orders of magnitude weaker than the EPR signal and (3) ESEM does not depend on a delicate balance of relaxation mechanisms as ENDOR does.

Although both, ESEM and $\mu \mathrm{SR}$, are time-domain techniques, only $\mu \mathrm{SR}$ itself is already a kinetic method. The paramagnetic species are created during the experiment and their fate is followed for the first few microseconds after their birth. Unlike ESEM, $\mu$ SR does not suffer from instrumental deadtime problems.

The versatility of $\mu \mathrm{SR}$ is, of course, restricted to the study of muonated species. Finally, it might be worthwhile to mention, that whereas ESEM- 
and ENDOR-measurements often require low temperatures, this is not the case for $\mu \mathrm{SR}$.

\section{Acknowledgements}

Thanks are due to Prof. H. Fischer and Prof. G. Lehmann for their continuous interest and support. Preprints from Prof. L. Kevan and Drs. D. R. Harshman, R. F. Kiefl and E. Roduner prior to publication are appreciated. I also wish to thank Dr. E. Roduner, who introduced me to the field of $\mu \mathrm{SR}$, for valuable discussions.

\section{References}

1. D. E. O'Reilly, Adv. Catal. 12 (1960) 31.

2. J. H. Lundsford, Adv. Catal. 22 (1972) 265.

3. B. D. Flockhart, in: Surface and Defect Properties of Solids, Chem. Soc. Spec. Period. Rep. 2 (1973) 69.

4. M. Ché, NATO ASI Ser. C61 (1980) 79.

5. R. F. Howe, Adv. Colloid Interface Sci. 18 (1982) 1.

6. E. G. Derouane and J. C. Vedrine, Ind. Chim. Belg. 38 (1973) 375.

7. J. H. Freed, ACS Symp. Ser. 34 (1976) 1.

8. C. C. Chao and J. H. Lundsford, J. Chem. Phys. 59 (1973) 3920.

9. L. Kevan and L. D. Kispert, Electron Spin Double Resonance Spectroscopy (Wiley, New York 1976).

10. M. M. Dorio and J. H. Freed, eds., Multiple Electron Resonance Spectroscopy (Plenum, New York 1979).

11. A. Schweiger, Struct. Bonding 51 (1982) 1.

12. W. B. Mims, in: Electron Paramagnetic Resonance, S. Geschwind, ed. (Plenum, New York 1972) chapt. 4.

13. L. Kevan and R. N. Schwartz, eds., Time Domaine Electron Spin Resonance (Wiley, New York 1979).

14. B. Webster, Ann. Rep. Prog. Chem. Sect. C81 (1984) 3.

15. L. Kevan and P. A. Narayana, in: Multiple Electron Resonance Spectroscopy, M. M. Dorio and J. H. Freed, eds. (Plenum, New York 1979) chapt. 6.

16. J. S. Hyde, in: Magnetic Resonance in Biological Systems, A. Ehrenberg, B. G. Malmström and T. Vänngard, eds. (Pergamon, Oxford 1967) p. 81.

17. G. H. Rist and J. S. Hyde, J. Chem. Phys. 52 (1970) 4633.

18. H. van Willingen and T. K. Chandrasekhar, J. Am. Chem. Soc. 105 (1983) 4232.

19. B. M. Hoffman, J. Martinsen and R. A. Venters, J. Magn. Reson. 59 (1984) 110.

20. N. D. Yordanov, M. Zdravkova and D. Shopov, Chem. Phys. Lett. 124 (1986) 191.

21. A. Schweiger, M. Rudin and J. Forrer, Chem. Phys. Lett. 80 (1981) 376.

22. R. B. Clarkson, NATO ASI Ser. C61 (1980) 425.

23. L. R. Dalton and A. L. Kwiram, J. Chem. Phys. 57 (1972) 1132.

24. A. Schweiger and Hs. H. Günthard, Mol. Phys. 42 (1981) 283.

25. A. Schweiger and Hs. H. Günthard, J. Magn. Reson. 57 (1984) 65.

26. J. S. Hyde, G. H. Rist and L. E. G. Eriksson, J. Phys. Chem. 72 (1968) 4269.

27. E. G. Derouane, Chem. Phys. Lett. 20 (1973) 269.

28. J. C. Vedrine, J. S. Hyde and D. S. Leniart, J. Phys. Chem. 76 (1972) 2087.

29. W. B. Mims, Phys. Rev. B5 (1972) 2049.

30. W. B. Mims, Phys. Rev. B6 (1972) 3543.

31. L. Kevan, M. K. Bowman, P. A. Narayana, R. K. Boekman, V. F. Yudanov and Yu. D. Tsvetkov, J. Chem. Phys. 63 (1975) 409. 
32. W. B. Mims, J. Peisach and J. L. Davies, J. Chem. Phys. 66 (1977) 5536.

33. M. Narayana, R. Y. Zhan and L. Kevan, J. Phys. Chem. 88 (1984) 3990.

34. A. Schweiger, L. Braunschweiler, J.-M. Fauth and R. R. Ernst, Phys. Rev. Lett. 54 (1985) 1241.

35. J. Michalik, M. Heming and L. Kevan, J. Phys. Chem. 90 (1986) 2132.

36. J.-M. Fauth, A. Schweiger, L. Braunschweiler and R. R. Ernst, J. Magn. Reson. 66 (1986) 74.

37. L. Kevan and M. Narayana, ACS Symp. Ser. 218 (1983) 283.

38. L. Kevan, to be published in Acc. Chem. Res., private communication.

39. G. L. Millhauser and J. H. Freed, J. Chem. Phys. 81 (1984) 37.

40. D. C. Walker, Muon and muonium chemistry (University Press, Cambridge 1983).

41. E. Roduner, Prog. React. Kinet. 14 (1986) 1.

42. P. Burkhard, E. Roduner, J. Hochmann and H. Fischer, J. Phys. Chem. 88 (1984) 773.

43. R. F. Kiefl, J. B. Warren, C. J. Oram, B. M. Marshall, J. H. Brewer, D. R. Harshman and C. W. Clawson, Phys. Rev. B 26 (1982) 2432.

44. R. F. Kiefl, Hyp. Inter. 8 (1981) 359.

45. D. R. Harshman, R. Keitel, M. Senba, R. F. Kiefl, E. J. Ansaldo and J. H. Brewer, Phys. Lett. 104A (1984) 472.

46. R. Keitel, M. Senba and D. R. Harshman, accepted for publication in Hyp. Inter.

47. R. F. Marzke, W. S. Glausinger, D. R. Harshman, E. J. Ansaldo, R. Keitel, M. Senba, D. R. Noakes, D. P. Spencer and J. H. Brewer, Chem. Phys. Lett. 120 (1985) 6.

48. R. F. Marzke, D. R. Harshman, E. J. Ansaldo, R. Keitel, M. Senba, D. R. Noakes and $\mathbf{J}$. H. Brewer, accepted for publication in Ultramicroscopy.

49. M. Heming, E. Roduner and B. D. Patterson, unpublished results.

50. P. W. Percival and H. Fischer, Chem. Phys. 16 (1976) 89.

51. A. Abragam, C. R. Acad. Sci. Ser. II, 299 (1984) 95.

52. R. F. Kiefl, S. Kreitzman, M. Celio, R. Keitel, G. M. Luke, J. H. Brewer, D. R. Noakes, P. W. Percival, T. Matsuzaki and K. Nishiyama, Phys. Rev. A 34 (1986) 100.

53. M. Heming, E. Roduner, B. D. Patterson, W. Odermatt, J. Schneider, Hp. Baumeler, H. Keller and I. M. Savić, Chem. Phys. Lett. 128 (1986) 100.

54. A. V. Astashkin, S. A. Dikanov and Yu. D. Tsvetkov, Chem. Phys. Lett. 122 (1985) 259.

55. A. de Groot, R. Evelo and A. J. Hoff, J. Magn. Reson. 66 (1986) 331.

56. E. Holzschuh, Phys. Rev. 27 B (1983) 102. 